\title{
Transcatheter Aortic Valve Implantation, Atrial Fibrillation, and Bleeding: A Surprisingly Fatal Attraction
}

\author{
James D. McFadyen ${ }^{1,2,3,4}$ Karlheinz Peter ${ }^{1,3,4,5}$ \\ ${ }^{1}$ Atherothrombosis and Vascular Biology Program, Baker Heart and \\ Diabetes Institute, Melbourne, Victoria, Australia \\ ${ }^{2}$ Department of Clinical Hematology, Alfred Hospital, Melbourne, \\ Victoria, Australia \\ ${ }^{3}$ Department of Medicine, Monash University, Melbourne, Victoria, \\ Australia \\ ${ }^{4}$ Department of Cardiometabolic Health, University of Melbourne, \\ Melbourne, Victoria, Australia \\ ${ }^{5}$ Department of Cardiology, Alfred Hospital, Melbourne, Victoria, \\ Australia
}

Thromb Haemost 2020;120:1479-1482.

The treatment of severe aortic stenosis (AS) has been transformed by the advent and widespread adoption of transcatheter aortic valve implantation (TAVI). Initially indicated for the management of severe AS in patients considered an unacceptably high surgical risk, there is now growing evidence that TAVI has a role in the management of AS across a spectrum of patients ranging from low to high surgical risk. ${ }^{1,2}$ The clinical importance of AS is ever increasing with the aging of the population, since the prevalence of AS exponentially increases with age such that approximately $10 \%$ of the population aged 80 to 89 have AS. $^{3}$ This rapidly evolving field has already ushered in new improvements regarding the application of advanced imaging modalities for preprocedure planning, the development of new generation devices, in addition to greater operator experience. ${ }^{4}$ These have led to improvements in TAVI safety and efficacy. Nevertheless, there remains an ongoing debate, and pressing clinical need, regarding the optimal antithrombotic strategy for patients undergoing TAVI given the Janus face of antithrombotic therapy helps prevent the high rates of ischemic stroke, yet endows a significant risk of bleeding complications. ${ }^{5}$ Indeed, while the optimal antithrombotic strategy for patients undergoing TAVI remains to be defined, it is sobering that the 1 year incidence of stroke is approximately 8 to $10 \%$, while the rate of bleeding approximates 30\% at 5 years, with similar proportions of access siteand nonaccess site-related bleeding. ${ }^{6-8}$ Current consensus guidelines (European Society of Cardiology and American College of Cardiology), recommend dual antiplatelet therapy (DAPT) with aspirin and clopidogrel for 3 to 6 months post-

received

September 7, 2020

accepted

September 8, 2020

Address for correspondence Karlheinz Peter, PhD, Atherothrombosis and Vascular Biology Program, Baker Heart and Diabetes Institute, 75 Commercial Road, P.O. Box 6492, Melbourne, VIC 3004, Australia (e-mail: karlheinz.peter@bakeridi.edu.au).

procedure, followed by long-term aspirin. ${ }^{1,9}$ However, the data supporting these recommendations remain limited, and are largely empiric, having been derived from recommendations for patients undergoing percutaneous coronary intervention (PCI). But notably, despite the larger extent of data available in the PCI population, the optimal antithrombotic strategy for elderly patients undergoing PCI similarly remains contentious given the elderly cohort represents a unique challenge given the association between advancing age and the heightened risk of both thrombosis and bleeding risk. ${ }^{10-12}$ Pleasingly, this important knowledge gap has begun to be addressed with the just recently published POPular TAVI trial demonstrating that aspirin, compared with DAPT for 3 months, for patients undergoing TAVI was associated with a significant reduction in bleeding rates (all bleeding events over 1 year: aspirin 15.1\%, aspirin + clopidogrel $26.6 \%, p=0.001$ ), without significant difference in the incidence of thromboembolic complications. ${ }^{13}$

A critical aspect regarding antithrombotic therapy postTAVI is the issue of patients with preexisting atrial fibrillation (AF). Indeed, up to $45 \%$ of high-risk patients undergoing TAVI have preexisting AF, and thus have a compelling indication for anticoagulation. ${ }^{14}$ As such, defining the optimal antithrombotic strategy that threads the needle of antithrombotic efficacy and safety in the TAVI patient cohort with $\mathrm{AF}$ is fundamentally important. This represents a major challenge as risk prediction both for thromboembolic and bleeding events in patients with AF, independent of TAVI, remains a challenge. ${ }^{15-17}$ In this context, the article in this issue by Lother et al sheds further light on the interplay

(c) 2020 Georg Thieme Verlag KG Stuttgart · New York
DOI https://doi.org/ 10.1055/s-0040-1718533. ISSN 0340-6245. 
between preexisting AF and outcomes in patients undergoing transfemoral TAVI (TF-TAVI). ${ }^{18}$ Using extensive registry data from over 55,000 patients, the authors demonstrate that in patients with AF undergoing TF-TAVI, bleeding was the strongest predictor of in-hospital mortality after adjustment for EuroSCORE and age (odds ratio [OR] 18.00, 95\% confidence interval $[\mathrm{CI}] 15.22-21.30, p<0.001)$. These findings are particularly striking given these data demonstrated that while stroke was also associated with increased mortality (OR 3.35, 95\% CI 2.61-4.30, $p<0.001$ ), this risk was significantly outweighed by the adverse prognostic effects of bleeding. Moreover, it is noteworthy that this study highlights the adverse effects of preexisting AF on TF-TAVI outcomes with this patient group displaying a 1.35 increase of in-hospital mortality compared with patients without AF.

While the data presented by Lother et $\mathrm{al}^{18}$ should be interpreted with usual caveats associated with a retrospective analysis, the reported findings are consistent with previous data emphasizing the prognostic significance of TAVI-associated bleeding. Indeed, early $(<30$ days $)$ major bleeding complications occur in 10 to $15 \%$ of patients undergoing TAVI, and are associated with adverse cardiovascular outcomes and increased mortality. ${ }^{19}$ Likewise, late bleeding complications are associated with a threefold increase in mortality. ${ }^{20}$ Against this back drop, it is significant that several studies now implicate AF as an adverse prognostic factor for patients undergoing TAVI. ${ }^{21}$ Although data regarding the specific antithrombotic therapy in the study by Lother et al was not available, it seems likely that more intense antithrombotic approaches incorporating anticoagulation in patients with AF is a central reason for the bleeding complications. However, whether AF, independent of antithrombotic regimen, confers an increased risk of bleeding in TAVI patients remains to be investigated.

The association of AS and bleeding ( - Fig. 1) has been well appreciated for over 50 years since the initial description of the association of calcific AS and gastrointestinal bleeding by Dr. E.C. Heyde. ${ }^{22}$ Indeed, Heyde's syndrome, as it became eponymously named, was later demonstrated to involve the intricate interplay between alterations in blood flow induced by the stenotic aortic valve, proteolysis of von Willebrand factor (vWF) high molecular weight multimers, and gastrointestinal angiodysplasia. ${ }^{23}$ More recently it has been elucidated that the high shear stress, imparted upon circulating $\mathrm{vWF}$ as it traverses the severely stenotic aortic valve, results in "unfolding" of vWF multimers making it more prone to a disintegrin and metalloproteinase with a thrombospondin type 1 motif, member 13 -mediated cleavage. ${ }^{24}$ While vWF multimers may normalize quickly post-TAVI, ${ }^{25}$ it is striking that recent reports have highlighted that this hemostatic abnormality may persist post-TAVI, even without significant post-TAVI paravalvular aortic regurgitation (PAVR). This raises the prospect that prosthetic valve design and/or placement may influence the hemostatic system. ${ }^{26}$ Importantly, there appears to be a direct link between hemostatic changes in AS and clinical outcomes, with a recent report detailing patients who underwent TAVI with DAPT and exhibited low platelet reactivity, had a nearly twofold in- crease in rates of bleeding. ${ }^{27}$ Moreover, a prolonged closure time with adenosine diphosphate using the point of care PFA-100 assay, is a strong predictor of TAVI-associated PAVR and also correlates with the risk of bleeding and mortality. ${ }^{28,29}$ As such, there appears a perilous balance to be struck between the requisite platelet inhibition to prevent thrombotic complications and the dreaded risk of bleeding. ${ }^{30,31}$ Indeed, the fact that current DAPT recommendations includes clopidogrel rather than more potent P2Y12 inhibitors, such as prasugrel and ticagrelor, reflects the caution toward stronger platelet inhibition and the associated higher bleeding risk, which is also reflected in the approach to use single antiplatelet therapy in TAVI patients deemed a high risk of bleeding. ${ }^{30,32}$

One aspect that surprisingly remains understudied in AS, is the effect of increased shear on blood cells passing through the stenotic aortic valve. Recently, it has been shown that monocytes, which play an important role in coagulation, exhibit an activated phenotype in AS patients which reverses post-TAVI. ${ }^{33}$ Although it is well accepted that platelets can be activated and desensitized by shear stress, there is a lack of data currently available regarding the effects of AS, and indeed TAVI, on platelet function. Therefore, mechanistic studies on the role of shear stress-induced effects on circulating blood cells will likely provide additional insights into the links between the potential prothrombotic effects and bleeding risk observed in patients undergoing TAVI.

Given the intimate relationship between severe AS and the hemostatic system, the implications of these findings presented by Lother et al are significant given the relative lack of randomized, controlled trials in the AF TAVI patient cohort. While current guidelines support the recommendation for concurrent oral anticoagulation (OAC) with either a direct oral anticoagulant or vitamin $\mathrm{K}$ antagonist (VKA) in addition to aspirin for patients with AF undergoing TAVI, there is a yearning need for prospective data to confirm such a therapeutic paradigm confers benefit. In this regard, recent data has challenged the benefit of antiplatelet therapy for patients already on VKAs, while highlighting such an approach is associated with a twofold risk of major and/or life-threatening bleeding. ${ }^{34}$ These findings have been reiterated by the recent POPular trial which investigated antithrombotic regimes for patients with a preexisting indication for anticoagulation (96\% AF) undergoing TAVI. Of note, patients that received anticoagulation alone had a significant reduction in bleeding complications when compared with those receiving OAC plus clopidogrel for 3 months (21.7\% vs. $34.6 \%, p=0.01){ }^{35}$ Thus, results from prospective trials such as the ENVISAGE-TAVI AF and AVATAR trials are eagerly awaited and will hopefully shed more light and provide further data on an optimal antithrombotic strategy. However, similar to the PCI domain, we may be observing the limits of our current antithrombotic strategies that have inherent limitations in their ability to straddle the risk of stroke, leaflet thrombosis, and bleeding. ${ }^{36}$ Therefore, in an era with an array of novel antithrombotic strategies in preclinical and early clinical development, the application of new antithrombotics may hold the ultimate promise for safer and more effective strategies for patients undergoing TAVI. ${ }^{36,37}$ 

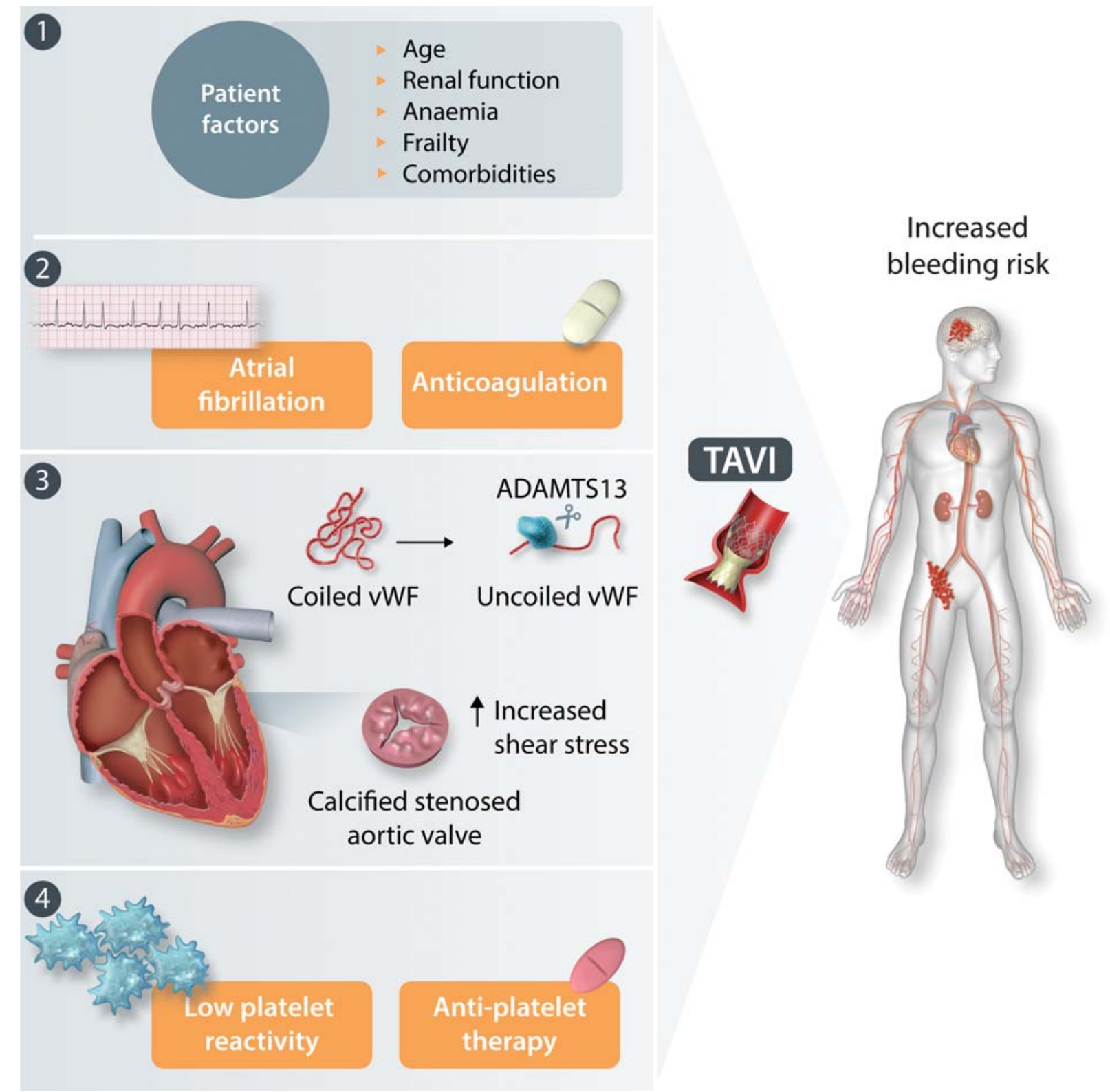

Fig. 1 Factors contributing to transcatheter aortic valve implantation (TAVI)-associated bleeding. The factors that contribute to TAVI-associated bleeding include: (1) patient factors including age, renal function, anemia, frailty, and comorbidities; (2) atrial fibrillation and the use of anticoagulation; (3) the development of acquired von Willebrand syndrome due to the effects of shear imparted on von Willebrand factor (vWF) at is traverses the stenotic aortic valve, which leads to a disintegrin and metalloproteinase with a thrombospondin type 1 motif, member 13 (ADAMTS13)-mediated proteolysis of vWF; and (4) low platelet reactivity and the use of antiplatelet therapy. These effects on the hemostatic system predispose patients undergoing TAVI to an increased risk of bleeding at both procedure-related and nonprocedure sites.

\section{Funding}

This study has been funded by the Department of Health, Australian Government, National Health and Medical Research Council, and National Heart Foundation of Australia.

\section{Conflict of Interest}

None declared.

\section{Acknowledgment}

We thank Eliana Stanziano for editorial assistance.

\section{References}

1 Baumgartner H, Falk V, Bax JJ, et al; ESC Scientific Document Group. 2017 ESC/EACTS Guidelines for the management of valvular heart disease. Eur Heart J 2017;38(36):2739-2791

2 Mack MJ, Leon MB, Thourani VH, et al; PARTNER 3 Investigators. Transcatheter aortic-valve replacement with a balloon-expandable valve in low-risk patients. N Engl J Med 2019;380(18):1695-1705

3 Ancona R, Pinto S. Epidemiology of aortic valve stenosis (AS) and of aortic valve incompetence (AI): is the prevalence of AS/AI similar in different parts of the world? e-J Cardiol Pract 2020;18:10

4 Rahhab Z, El Faquir N, Tchetche D, et al. Expanding the indications for transcatheter aortic valve implantation. Nat Rev Cardiol 2020; 17(02):75-84 
5 Ranasinghe MP, Peter K, McFadyen JD. Thromboembolic and bleeding complications in transcatheter aortic valve implantation: insights on mechanisms, prophylaxis and therapy. J Clin Med 2019;8(02):280

6 Vranckx P, Windecker S, Welsh RC, Valgimigli M, Mehran R, Dangas G. Thrombo-embolic prevention after transcatheter aortic valve implantation. Eur Heart J 2017;38(45):3341-3350

7 Konigstein M, Ben-Assa E, Banai S, et al. Periprocedural bleeding, acute kidney injury, and long-term mortality after transcatheter aortic valve implantation. Can J Cardiol 2015;31(01):56-62

8 Piccolo R, Pilgrim T, Franzone A, et al. Frequency, timing, and impact of access-site and non-access-site bleeding on mortality among patients undergoing transcatheter aortic valve replacement. JACC Cardiovasc Interv 2017;10(14):1436-1446

9 Nishimura RA, Otto CM, Bonow RO, et al. 2017 AHA/ACC focused update of the 2014 AHA/ACC guideline for the management of patients with valvular heart disease: a report of the American College of Cardiology/American Heart Association task force on clinical practice guidelines. Circulation 2017;135(25):e1159-e1195

10 Capodanno D, Greco A. Risk stratification for bleeding in the elderly with acute coronary syndrome: not so simple. Thromb Haemost 2018;118(06):949-952

11 Sorrentino S, Baber U, Claessen BE, et al. Determinants of significant out-of-hospital bleeding in patients undergoing percutaneous coronary intervention. Thromb Haemost 2018;118(11):1997-2005

12 Schäfer A, Flierl U, Bauersachs J. Anti-thrombotic strategies in elderly patients receiving platelet inhibitors. Eur Heart J Cardiovasc Pharmacother 2020;6(01):57-68

13 Brouwer J, Nijenhuis VJ, Delewi R, et al. Aspirin with or without clopidogrel after transcatheter aortic-valve implantation. $\mathrm{N}$ Engl J Med 2020; e-published ahead of print

14 Stachon P, Kaier K, Zirlik A, et al. Risk-adjusted comparison of inhospital outcomes of transcatheter and surgical aortic valve replacement. J Am Heart Assoc 2019;8(07):e011504

15 Borre ED, Goode A, Raitz G, et al. Predicting thromboembolic and bleeding event risk in patients with non-valvular atrial fibrillation: a systematic review. Thromb Haemost 2018;118(12):2171-2187

16 Proietti M, Mujovic N, Potpara TS. Optimizing stroke and bleeding risk assessment in patients with atrial fibrillation: a balance of evidence, practicality and precision. Thromb Haemost 2018;118 (12):2014-2017

17 Edmiston MK, Lewis WR. Bleeding risk scores in atrial fibrillation: helpful or harmful? J Am Heart Assoc 2018;7(18):e010582

18 Lother A, Kaier K, Ahrens I, et al. Bleeding complications drive inhospital mortality of patients with atrial fibrillation after transcatheter aortic valve replacement. Thromb Haemost 2020;120 (11):1580-1586

19 Généreux P, Cohen DJ, Williams MR, et al. Bleeding complications after surgical aortic valve replacement compared with transcatheter aortic valve replacement: insights from the PARTNER I Trial (Placement of Aortic Transcatheter Valve). J Am Coll Cardiol 2014;63(11):1100-1109

20 Généreux P, Cohen DJ, Mack M, et al. Incidence, predictors, and prognostic impact of late bleeding complications after transcatheter aortic valve replacement. J Am Coll Cardiol 2014;64(24):2605-2615
21 Tarantini G, Mojoli M, Urena M, Vahanian A. Atrial fibrillation in patients undergoing transcatheter aortic valve implantation: epidemiology, timing, predictors, and outcome. Eur Heart J 2017;38(17):1285-1293

22 Heyde E. Gastrointestinal bleeding in aortic stenosis. N Engl J Med 1958;259:196

23 Loscalzo J. From clinical observation to mechanism-Heyde's syndrome. N Engl J Med 2012;367(20):1954-1956

24 Van Belle E, Rauch A, Vincentelli A, et al. Von Willebrand factor as a biological sensor of blood flow to monitor percutaneous aortic valve interventions. Circ Res 2015;116(07):1193-1201

25 Van Belle E, Rauch A, Vincent F, et al. von Willebrand factor multimers during transcatheter aortic-valve replacement. $\mathrm{N}$ Engl J Med 2016;375(04):335-344

26 Pawlitschek F, Keyl C, Zieger B, et al. Alteration of von Willebrand factor after transcatheter aortic valve replacement in the absence of paravalvular regurgitation. Thromb Haemost 2018;118(01): 103-111

27 Gross L, Jochheim D, Nitschke T, et al. Platelet reactivity and early outcomes after transfemoral aortic valve implantation. Thromb Haemost 2018;118(10):1832-1838

28 Kibler M, Marchandot B, Messas N, et al. CT-ADP point-of-care assay predicts 30-day paravalvular aortic regurgitation and bleeding events following transcatheter aortic valve replacement. Thromb Haemost 2018;118(05):893-905

29 Casonato A, Galletta E, Cella G, Barbon G, Daidone V. Acquired von Willebrand syndrome hiding inherited von Willebrand disease can explain severe bleeding in patients with aortic stenosis. Arterioscler Thromb Vasc Biol 2020;40(09):2187-2194

30 Capodanno D, Greco A. Platelet function testing after transcatheter aortic valve implantation. Thromb Haemost 2018;118(10): $1681-1685$

31 Trejo-Velasco B, Cruz-González I, Tello-Montoliu A, et al. Influence of valve type and antiplatelet regimen on platelet reactivity after TAVI: subanalysis of the REAC-TAVI trial. J Invasive Cardiol 2020: JIC20200810-2

32 Greco A, Capodanno D. Anticoagulation after transcatheter aortic valve implantation: current status. Interv Cardiol (Lond) 2020;15:e02

33 Baratchi S, Zaldivia MTK, Wallert M, et al. TAVI represents an antiinflammatory therapy via reduction of shear stress induced, Piezo-1-mediated monocyte activation. Circulation 2020;142 (11):1092-1105

34 Abdul-Jawad Altisent O, Durand E, Muñoz-García AJ, et al. Warfarin and antiplatelet therapy versus warfarin alone for treating patients with atrial fibrillation undergoing transcatheter aortic valve replacement. JACC Cardiovasc Interv 2016;9(16):1706-1717

35 Nijenhuis VJ, Brouwer J, Delewi R, et al. Anticoagulation with or without clopidogrel after transcatheter aortic-valve implantation. N Engl J Med 2020;382(18):1696-1707

36 McFadyen JD, Schaff M, Peter K. Current and future antiplatelet therapies: emphasis on preserving haemostasis. Nat Rev Cardiol 2018;15(03):181-191

37 McFadyen JD, Peter K. Novel antithrombotic drugs on the horizon: the ultimate promise to prevent clotting while avoiding bleeding. Circ Res 2017;121(10):1133-1135 